Supplementary Material for:

\title{
Chemical Compatibility of Battery Electrolytes with Rapid Prototyping Materials and Adhesives
}

Sophia E. Lee, Oliver C. Harris, An Dinh Song Nguyen, Maureen H. Tang*

Department of Chemical and Biological Engineering, Drexel University, 3141 Chestnut St, Philadelphia, PA 19104, United States

*Corresponding Author: mhtang@drexel.edu 
Table S1 Summary of physical changes and chemical compatibility for all materials and solvents

\begin{tabular}{|c|c|c|c|c|c|}
\hline $\mathrm{PC}$ & Grade & Hardness & Deformation & Fracture & Note \\
\hline Resin (green) & A & - & - & - & - \\
\hline Resin (90 min) & A & - & - & - & - \\
\hline Hysol 9462 & $\mathrm{~B}$ & - & - & - & - \\
\hline Lord 406/19 & $\mathrm{C}$ & 1 week & - & - & - \\
\hline MarineWeld & $\mathrm{B}$ & - & - & - & - \\
\hline Gorilla Glue & $\mathrm{C}$ & - & - & 1-4 week & - \\
\hline Devcon & $\mathrm{B}$ & - & - & - & - \\
\hline Viton & $\mathrm{D}$ & - & 24 hours & 24 hours & $\mathrm{F}$ \\
\hline EC: DMC & Grade & Hardness & Deformation & Fracture & Note \\
\hline Resin (green) & $\mathrm{B}$ & - & - & 4 weeks & - \\
\hline Resin (30 min) & $\mathrm{A}$ & - & - & - & - \\
\hline Resin (90 min) & $\mathrm{A}$ & - & - & - & - \\
\hline Hysol 9462 & $\mathrm{~B}$ & 1-4 weeks & - & - & - \\
\hline Lord 406/19 & $\mathrm{C}$ & 24 hours & - & - & - \\
\hline MarineWeld & $\mathrm{C}$ & - & - & 4 weeks & - \\
\hline Gorilla Glue & $\mathrm{D}$ & - & - & 1-7 days & $\mathrm{F}$ \\
\hline Devcon & $\mathrm{C}$ & - & - & - & - \\
\hline Viton & $\mathrm{D}$ & - & 24 hours & 24 hours & $\mathrm{F}$ \\
\hline DME & Grade & Hardness & Deformation & Fracture & Note \\
\hline Resin (green) & $\mathrm{D}$ & - & 24 hours & - & $\mathrm{F}$ \\
\hline Resin (30 min) & $\mathrm{B}$ & - & - & 4 weeks & - \\
\hline Resin (90 min) & $\mathrm{B}$ & - & - & 4 weeks; minor & - \\
\hline Hysol 9462 & $\mathrm{~B}$ & - & - & - & - \\
\hline Lord 406/19 & $\mathrm{C}$ & - & - & - & - \\
\hline MarineWeld & $\mathrm{B}$ & - & - & - & - \\
\hline Gorilla Glue & $\mathrm{D}$ & - & - & 24 hours & $\mathrm{F}$ \\
\hline Devcon & $\mathrm{D}$ & 24 hours & - & 24 hours & $\mathrm{F}$ \\
\hline Viton & $\mathrm{C}$ & - & 1 week & 1 week & $\mathrm{F}$ \\
\hline $\mathrm{MeCN}$ & Grade & Hardness & Deformation & Fracture & Note \\
\hline Resin (green) & $\mathrm{D}$ & - & 24 hours & - & $\mathrm{F}$ \\
\hline Resin (30 min) & $\mathrm{C}$ & - & - & 1 month & - \\
\hline Resin (90 min) & $\mathrm{B} / \mathrm{C}$ & - & - & 1 month & - \\
\hline Hysol 9462 & $\mathrm{~B}$ & 1 week; minor & - & - & - \\
\hline Lord 406/19 & $\mathrm{C}$ & - & - & - & - \\
\hline MarineWeld & $\mathrm{A}$ & - & - & - & - \\
\hline Gorilla Glue & $\mathrm{C}$ & - & - & 1 week & $\mathrm{F}$ \\
\hline Devcon & $\mathrm{D}$ & 24 hours & - & 24 hours & $\mathrm{F}$ \\
\hline Viton & $\mathrm{C}$ & - & 1 week & 1 week & $\mathrm{F}$ \\
\hline LP 30 & Grade & Hardness & Deformation & Fracture & Note \\
\hline Resin (90 min) & A & - & - & - & - \\
\hline Hysol & $\mathrm{C}$ & 1 week & - & 1 week & $\mathrm{F}$ \\
\hline Lord & $\mathrm{C}$ & 4 weeks & - & - & - \\
\hline
\end{tabular}




\begin{tabular}{|l|lllll}
\hline MarineWeld & C & - & - & 4 weeks & - \\
\hline $1 \mathrm{M} \mathrm{NaClO}_{4} / \mathrm{PC}$ & Grade & Hardness & Deformation & Fracture & Note \\
\hline Resin $(90 \mathrm{~min})$ & A & - & - & - & - \\
\hline Hysol 9462 & B & - & - & - & - \\
\hline Lord 406/19 & C & 24 hours & - & - & - \\
\hline MarineWeld & C & 4 weeks & - & - & - \\
\hline
\end{tabular}

Grades: $\mathrm{A}=$ less than $2 \%$ change over 1 month, no significant change in properties, $\mathrm{B}=2-10 \%$ change over 1 month, with mild to no mechanical degradation, $\mathrm{C}=$ greater than $10 \%$ change and/or mechanical degradation in 1-4 weeks, $\mathrm{D}=$ Significant changes or failure within 24 hours. Notes: $\mathrm{F}$ = Failure; dissolution in solvent or mechanical instability preventing further testing 